\title{
Hilbert Boundary Value Problem with an Unknown Function on Arbitrary Infinite Straight Line
}

\author{
Lixia Cao \\ Mathematics College, Northeast Petroleum University, Daqing, China \\ Email: caolixia98237@163.com
}

Received December 9, 2012; revised January 11, 2013; accepted January 29, 2013

\begin{abstract}
We consider a Hilbert boundary value problem with an unknown parametric function on arbitrary infinite straight line passing through the origin. We propose to transform the Hilbert boundary value problem to Riemann boundary value problem, and address it by defining symmetric extension for holomorphic functions about an arbitrary straight line passing through the origin. Finally, we develop the general solution and the solvable conditions for the Hilbert boundary value problem.
\end{abstract}

Keywords: Arbitrary Infinite Straight Line; Symmetric Extension; Hilbert Boundary Value Problem; Unknown Function; Riemann Boundary Value Problem

\section{Introduction}

Various kinds of boundary value problems (BVPs) for analytic functions or polyanalytic functions have been widely investigated [1-8]. The main approach is to use the decomposition of polyanalytic functions and their generalization to transform the boundary value problems to their corresponding boundary value problems for analytic functions. Recently, inverse Riemann BVPs for generalized holomorphic functions or bianalytic functions have been investigated [9-12].

In this paper, we consider a kind of Hilbert BVP with an unknown parametric function. We first define the symmetric extension of holomorphic function about an infinite straight line passing through the origin, and discuss its several important properties. And after, we propose a Hilbert BVP with an unknown parametric function on arbitrary half-plane with its boundary passing through the origin. Then, we transform the Hilbert BVP into a Riemann BVP on the infinite straight line using the defined symmetric extension. Finally, we discuss the solvable conditions and the solution for the Hilbert BVP.

\section{A Hilbert Boundary Value Problem with an Unknown Function}

Let $L$ be an infinite straight line with an inclination $\theta(0<\theta<\pi)$ in the complex plane, passing through the origin and being oriented in upward direction. Let $L^{+}$ and $L^{-}$denote the upper half-plane and the lower halfplane cut by $L$.

Our objective is to find a pair of functions
$\{\Phi(z), \Psi(t)\}$, where $\Phi(z)$ is holomorphic in the domain $L^{+}$and continuously extendable to its boundary $L$, and $\Psi(t) \in \hat{H}(L)$ is real-valued and Holder continuous on $L$, satisfying the following boundary conditions

$$
\left\{\begin{array}{l}
\operatorname{Re}\left[\omega_{1}(t) \Phi^{+}(t)\right]=g_{1}(t) \Psi(t) / 2+c_{1}(t) / 2, \\
\operatorname{Re}\left[\omega_{2}(t) \Phi^{+}(t)\right]=g_{2}(t) \Psi(t) / 2+c_{2}(t) / 2,
\end{array} t \in L,\right.
$$

where

$$
\omega_{j}(t)=a_{j}(t)+\mathrm{i} b_{j}(t)(j=1,2),
$$

and

$$
a_{j}(t), b_{j}(t), c_{j}(t) \in \hat{H}(L)(j=1,2)
$$

are given functions.

\section{Symmetric Extension of Holomorphic Functions about an Infinite Straight Line}

An important step in solving problem (1) is to define a symmetric extension of holomorphic functions about the infinite straight line $L$ with an inclination $\theta(0<\theta<\pi)$.

For a holomorphic function $\Phi(z)$ in the simplyconnected domain $L^{+}$, we define the symmetric extension of $\Phi(z)$ about $L$ as follows:

$$
\Phi_{*}(z)=\mathrm{e}^{2 \mathrm{i} \theta} \overline{\Phi(\tilde{z})},
$$

where $\tilde{z}=\bar{z} \mathrm{e}^{2 \mathrm{i} \theta}$ is the symmetric point of $z$ about $L$. For simplicity, we express $\Phi_{*}(z)$ as $\tilde{\Phi}(z)$. From 
definition (2), we may establish that

1) $\Phi_{* *}(z)=\Phi(z)$;

2) If $\Phi(z)$ is defined in $L^{+}+L^{-}$, then $\Phi_{*}(z)$ is also defined in $L^{+}+L^{-}$;

3) If $\Phi(z)$ is holomorphic in $L^{+}$, then $\Phi_{*}(z)$ is holomorphic in $L^{-}$because of

$$
\begin{aligned}
\frac{\mathrm{d} \Phi_{*}(z)}{\mathrm{d} z} & =\mathrm{d}\left[\overline{\Phi(\tilde{z})} \mathrm{e}^{2 \mathrm{i} \theta}\right] / \mathrm{d} z=\left[\mathrm{d} \overline{\Phi(\tilde{z})} \mathrm{e}^{2 \mathrm{i} \theta}\right] /\left[\mathrm{e}^{2 \mathrm{i} \theta} \mathrm{d} \overline{\tilde{z}}\right] \\
& =\mathrm{d} \overline{\Phi(\tilde{z})} / \mathrm{d} \overline{\tilde{z}}=\overline{(\mathrm{d} \Phi(\tilde{z}) / \mathrm{d} \tilde{z})}
\end{aligned}
$$

4) If a holomorphic $\Phi(z)$ in $L^{+}$can be continuously extended to $L$, then $\Phi_{*}(z)$ in $L^{-}$can be continuously extended to $L$, and their boundary value on $L$ satisfies the following equality

$$
\Phi_{*}^{-}(t)=\mathrm{e}^{2 \mathrm{i} \theta} \overline{\Phi^{+}(t)} ;
$$

5) If $\Phi(z)$ is holomorphic in $L^{+}$and continuous on $L^{+}+L$, then

$$
\Omega(z)=\left\{\begin{array}{l}
\Phi(z), z \in L^{+}, \\
\Phi_{*}(z), z \in L^{-}
\end{array}\right.
$$

is a sectionally holomorphic function that jumps on $L$ with $\Omega(\infty)$ finite, and $\Omega(z)$ possesses the following properties:

$$
\begin{gathered}
\Omega^{-}(t)=\mathrm{e}^{2 \mathrm{i} \theta} \overline{\Omega^{+}(t)}, t \in L \\
\Omega_{*}(z)=\Omega(z), z \in L^{+} \cup L^{-} \\
\Omega_{*}^{+}(t)=\mathrm{e}^{2 \mathrm{i} \theta} \overline{\Omega^{-}(t)}, \Omega_{*}^{-}(t)=\mathrm{e}^{2 \mathrm{i} \theta} \overline{\Omega^{+}(t)} .
\end{gathered}
$$

6) Let $h(z)=f(z) \cdot g(z)$, where $f(z)$ and $g(z)$ are holomorphic in $L^{+}\left(L^{-}\right.$or $\left.L^{+}+L^{-}\right)$. It is not necessarily true that $h_{*}(z)=f_{*}(z) \cdot g_{*}(z)$.

Problem (1) is normal only if $\alpha(t) \neq 0$ on $L$.

\section{Transformation of Problem (1)}

In this section, we develop a general method to solve boundary value problem (1) or similar problems. Let

$$
\begin{aligned}
& \alpha(t)=\omega_{1}(t) g_{2}(t)-\omega_{2}(t) g_{1}(t) \\
& \beta(t)=c_{1}(t) g_{2}(t)-c_{2}(t) g_{1}(t) .
\end{aligned}
$$

Multiplying the first and the second equation in (1) by $g_{2}(t)$ and $g_{1}(t)$ respectively, we obtain the Riemann boundary problem

$$
\operatorname{Re}\left[\alpha(t) \Phi^{+}(t)\right]=\beta(t) / 2, t \in L
$$

or

$$
\alpha(t) \Phi^{+}(t)+\overline{\alpha(t)} \mathrm{e}^{-2 i \theta} \overline{\mathrm{e}^{-2 i \theta} \Phi^{+}(t)}=\beta(t), t \in L .
$$

By extending $\Phi(z)$ to $L^{-}$about the straight line $L$, we obtain a sectionally holomorphic function $\Omega(z)$ as
(4) with jump $L$, satisfying the boundary conditions

$$
\Omega^{+}(t)=\Phi^{+}(t), \Omega^{-}(t)=\overline{\mathrm{e}^{-2 \mathrm{i} \theta} \Phi^{+}(t)}, t \in L .
$$

Thus (9) can be rewritten in the form

$$
\alpha(t) \Omega^{+}(t)+\overline{\alpha(t)} \mathrm{e}^{-2 i \theta} \Omega^{-}(t)=\beta(t), t \in L .
$$

Due to $\alpha(t) \neq 0,(10)$ can be written as R problem

$$
\Omega^{+}(t)=G(t) \Omega^{-}(t)+g(t), t \in L,
$$

where

$$
G(t)=-\mathrm{e}^{-2 \mathrm{i} \theta} \overline{\alpha(t)} / \alpha(t), g(t)=\beta(t) / \alpha(t)
$$

and $G(t), g(t) \in \widehat{H}(L), G(t) \neq 0$ on $L$.

If $\Phi(z)$ is a solution of problem (1), then $\Omega(z)$ extended from $\Phi(z)$ by (4) must be a solution of (10) or (10)' in $R_{0}$ (namely $\Omega(\infty)=0$ ) and satisfies the boundary condition (5). On the other hand, if the solution $\Omega(z)$ of R problem (10)' in $R_{0}$ satisfies the boundary condition (5), then $\Omega^{+}(z)=\Phi(z)$ is really a solution of problem (1). Consequently, problem (1) is equivalent to $\mathrm{R}$ problem (10)' in $R_{0}$ together with the additive condition (5).

Assume that $\Omega(z)$ is a solution of (10) in $R_{0}$, by making conjugate for (10) we obtain

$$
\overline{\alpha(t)} \overline{\Omega^{+}(t)}+\alpha(t) \mathrm{e}^{2 \mathrm{i} \theta} \overline{\Omega^{-}(t)}=\beta(t), t \in L .
$$

We read from relation (7) that $\Omega_{*}(z)$ is also a solution of (10)' in $R_{0}$, so that

$$
\Omega_{0}(z)=\left[\Omega(z)+\Omega_{*}(z)\right] / 2
$$

is a solution of (10)' in class $R_{0}$ and satisfies the additive condition (5). So that, whenever we find out the solution $\Omega(z)$ of problem (10)' in class $R_{0}$, and write out $\Omega_{*}(z)$, then

$$
\Omega_{0}(z)=\left[\Omega(z)+\Omega_{*}(z)\right] / 2
$$

is actually the solution of problem (1).

Let

$$
\kappa=\frac{1}{2 \pi}[\arg G(t)]_{L}=\frac{1}{\pi}[\arg \alpha(t)]_{L} .
$$

By $a_{j}(t), b_{j}(t), c_{j}(t) \in \hat{H}(L)$ we know that $\kappa$ is even.

\section{Solution of the Hilbert Boundary Value Problem with an Unknown Function}

Here, we only consider the problem (1) in the normal case. The nonnormal case can be solved similarly.

\subsection{Homogeneous Problem}

The homogeneous problem of (1) is as follows 


$$
\left\{\begin{array}{l}
\operatorname{Re}\left[\omega_{1}(t) \Phi^{+}(t)\right]=g_{1}(t) \Psi(t) / 2, \\
\operatorname{Re}\left[\omega_{2}(t) \Phi^{+}(t)\right]=g_{2}(t) \Psi(t) / 2,
\end{array} t \in L .\right.
$$

By canceling the unknown function $\Psi(t)$, problem (12) becomes

$$
\operatorname{Re}\left[\left(\omega_{1} g_{2}-\omega_{2} g_{1}\right) \Phi^{+}\right]=0, t \in L,
$$

which corresponds to the homogeneous problem of $\mathrm{R}$ problem (10)'

$$
\Omega^{+}(t)=G(t) \Omega^{-}(t), t \in L .
$$

Setting $z_{0}=\mathrm{ie}^{\mathrm{i} \theta} \in L^{+}$, we have

$$
\tilde{z}_{0}=\bar{z}_{0} \mathrm{e}^{2 \mathrm{i} \theta}=-\mathrm{e}^{\mathrm{i} \theta} \in L^{-} .
$$

If we let $G_{0}(t)=G(t)\left(t-\tilde{z}_{0}\right)^{\kappa} /\left(t-z_{0}\right)^{\kappa}$, then we know $G_{0}(t) \in \hat{H}(L), G_{0}(t) \neq 0$ on $L$ with $G_{0}(\infty)=G(\infty) \neq 0$, and $\operatorname{Ind}_{L} G_{0}(t)=0$. By letting

$$
V(z)=\left\{\begin{array}{l}
\left(z-\tilde{z}_{0}\right)^{\kappa} \Omega(z), z \in L^{+}, \\
\left(z-z_{0}\right)^{\kappa} \Omega(z), z \in L^{-},
\end{array}\right.
$$

Let us introduce the function

$$
\Gamma(z)=\frac{1}{2 \pi \mathrm{i}} \int_{L} \frac{\log G_{0}(t)}{t-z} \mathrm{~d} t, z \notin L .
$$

Since $\left|G_{0}(t)\right| \equiv 1$, we have

$$
\Gamma(z)=\frac{1}{2 \pi} \int_{L} \frac{\Theta(t)}{t-z} \mathrm{~d} t, z \notin L,
$$

where

$$
\Theta(t)=\arg \left\{-\left(t+\tilde{z}_{0}\right)^{2 \kappa}\left[\omega_{1}(t) g_{2}(t)-\omega_{2}(t) g_{1}(t)\right]^{2}\right\}
$$

is real-valued on $L$ and $\Gamma(\infty)=0$. Now the canonical function of R problem (15) or (14) can be taken as

$$
X(z)=\left\{\begin{array}{l}
A\left(z-\tilde{z}_{0}\right)^{-\kappa} \mathrm{e}^{\Gamma(z)}, z \in L^{+}, \\
A\left(z-z_{0}\right)^{-\kappa} \mathrm{e}^{\Gamma(z)}, z \in L^{-},
\end{array}\right.
$$

where $A$ is an unknown complex constant. We can see from (17) that $X^{+}(t) / X^{-}(t)=G(t)$, thus $\mathrm{R}$ problem (15) can be transferred to the following problem

$$
\Omega^{+}(t) / X^{+}(t)=\Omega^{-}(t) / X^{-}(t), t \in L .
$$

So $F(z)=\Omega(z) / X(z)$ is holomorphic on the whole complex plane and has $\kappa$ order at $\infty$. From [5] we know that the general solution of R problem (14) in $R_{0}$ takes the form

$$
\Omega(z)=X(z) P_{\kappa}(z),
$$

where $P_{\kappa}(z)=c_{0} z^{\kappa}+c_{1} z^{\kappa-1}+\cdots+c_{\kappa}$ is an arbitrary polynomial of degree $\kappa$ with $P_{\kappa}(z) \equiv 0$ if $\kappa<0$.

According to (16), we know

$$
\overline{\Gamma(\tilde{z})}=\Gamma(z)
$$

and hence

$$
\overline{\Gamma^{+}(t)}=\Gamma^{-}(t) .
$$

From (17) and (20) it can be seen that

$$
\tilde{X}(z)=\left\{\begin{array}{l}
\overline{X(\tilde{z})} \mathrm{e}^{2 \mathrm{i} \theta}=\bar{A} \mathrm{e}^{2 \mathrm{i} \theta(\kappa+1)}\left(z-\tilde{z}_{0}\right)^{-\kappa} \mathrm{e}^{\Gamma(z)}, z \in L^{+}, \\
\mathrm{e}^{2 \mathrm{i} \theta} \overline{X(\tilde{z})}=\bar{A} \mathrm{e}^{2 \mathrm{i} \theta(\kappa+1)}\left(z-z_{0}\right)^{-\kappa} \mathrm{e}^{\Gamma(z)}, z \in L^{-},
\end{array}\right.
$$

which implies that $\tilde{X}(z)=\frac{\bar{A}}{A} \mathrm{e}^{2(\kappa+1) i \theta} X(z)$. By taking

$$
A=\mathrm{e}^{\mathrm{i} \theta(\kappa+1)}
$$

we obtain

$$
\tilde{X}(z)=X(z)
$$

and

$$
\begin{aligned}
& \Omega_{*}(z) \\
& =\mathrm{e}^{2 \mathrm{i} \theta} \overline{\Omega(\tilde{z})}=\mathrm{e}^{2 \mathrm{i} \theta} \overline{X(\tilde{z})} \overline{P_{\kappa}(\tilde{z})} \\
& =X(\mathrm{z})\left[\overline{c_{0}} \mathrm{e}^{-2 \kappa \theta \mathrm{i}} \mathrm{z}^{\kappa}+\overline{{C_{1}}} \mathrm{e}^{-2(\kappa-1) \theta \mathrm{i}} \mathrm{z}^{\kappa-1}+\cdots+\overline{c_{\kappa}}\right] .
\end{aligned}
$$
if

Consequently, we see that $\Omega_{*}(z)=\Omega(z)$ if and only

$$
c_{0}=\overline{c_{0}} \mathrm{e}^{-2 \kappa \theta \mathrm{i}}, c_{1}=\overline{c_{1}} \mathrm{e}^{-2(\kappa-1) \theta \mathrm{i}}, c_{2}=\overline{c_{2}} \mathrm{e}^{-2(\kappa-2) \theta \mathrm{i}}, \cdots, c_{\kappa}=\overline{c_{\kappa}} .
$$

Then when condition (24) is satisfied, the solution of $\mathrm{H}$ problem (13) is given by (19).

Now putting the solution $\Phi(z)$ of $\mathrm{H}$ problem (13) given by (19) into the first equation (or the second equation) in (12), we get

$$
\Psi(t)=\left\{2 \operatorname{Re}\left[\omega_{1}(t) \Phi^{+}(t)\right]\right\} / g_{1}(t), t \in L .
$$

Thus we get the following results.

Theorem 5.1. For the homogeneous problem (12), the following two cases arise.

1) When $\kappa \geq 0$, its general solution is $(\Phi(z), \Psi(t))$, where $\Phi(z)$ and $\Psi(t)$ are given by (19) and (25) respectively, in which condition (24) is satisfied for $P_{\kappa}(z)$, and $A$ is given by (22) (a real constant factor is permitted for $A$ ).

2) When $\kappa<0$, it only has zero-solution

$$
(\Phi(z), \Psi(t))=(0,0) .
$$

\subsection{Nonhomogeneous Problem}

In order to solve the n nonhomogeneous problem (1), we 
only need to find out a particular solution for problem (1).

According to [5], we know that when $\kappa \geq 0$ the $\mathrm{R}$ problem (10)' a particular solution in class $R_{0}$ as follows

$$
U(z)=\frac{Y(z)}{2 \pi \mathrm{i}} \int_{L} \frac{\left(c_{1} g_{2}-c_{2} g_{1}\right) \mathrm{d} t}{\left(\omega_{1} g_{2}-\omega_{2} g_{1}\right) Y^{+}(t)(t-z)}, z \notin L .
$$

Therefore $\Phi_{0}(z)=\left[U(z)+U_{*}(z)\right] / 2$ is actually the particular solution of problem (1), where

$$
\begin{aligned}
Y(z) & =\left(z-\tilde{z}_{0}\right)^{\kappa} X(z) \\
& =\left\{\begin{array}{l}
A \mathrm{e}^{\Gamma(z)}, z \in L^{+}, \\
A \mathrm{e}^{\Gamma(z)}\left(z-\tilde{z}_{0}\right)^{\kappa} /\left(z-z_{0}\right)^{\kappa}, z \in L^{-} .
\end{array}\right.
\end{aligned}
$$

And from (20) we obtain

$$
Y_{*}(z)=\frac{\bar{A}}{A} \mathrm{e}^{2 \mathrm{i} \theta} Y(z)\left(z-z_{0}\right)^{\kappa} /\left(z-\tilde{z}_{0}\right)^{\kappa}, z \notin L .
$$

It follows from (3) that $Y_{*}^{-}(t)=\mathrm{e}^{2 \mathrm{i} \theta} \overline{Y^{+}(t)}$, and from (20) and (21) that

$$
Y_{*}^{-}(t)=\frac{\bar{A}}{A} Y^{-}(t) \mathrm{e}^{2 \mathrm{i} \theta}\left(t-z_{0}\right)^{\kappa} /\left(t-\tilde{z}_{0}\right)^{\kappa}, t \in L .
$$

While due to $X^{+}(t)=G(t) X^{-}(t)$ and (28) we have $Y^{+}(t)=G(t) Y^{-}(t)$, so we obtain

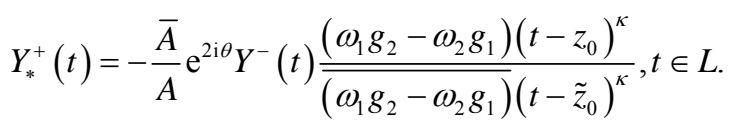

Therefore, we obtain

$$
U_{*}(z)=-\left(\frac{z-z_{0}}{z-\tilde{z}_{0}}\right)^{\kappa} \frac{Y(z)}{2 \pi \mathrm{i}} \int_{L} \frac{\left(\frac{t-\tilde{z}_{0}}{t-z_{0}}\right)^{\kappa}\left(c_{1} g_{2}-c_{2} g_{1}\right) \mathrm{d} t}{\left(\omega_{1} g_{2}-\omega_{2} g_{1}\right) Y^{+}(t)(t-z)},
$$$$
Z \notin L
$$

and

$$
\begin{aligned}
\Phi_{0}(z)= & \frac{Y(z)}{4 \pi \mathrm{i}} \int_{L} \frac{\left(c_{1} g_{2}-c_{2} g_{1}\right) \mathrm{d} t}{\left(\omega_{1} g_{2}-\omega_{2} g_{1}\right) Y^{+}(t)(t-z)} \\
& +\frac{Y(z)}{4 \pi \mathrm{i}}\left(\frac{z-z_{0}}{z-\tilde{z}_{0}}\right)^{\kappa} \int_{L} \frac{\left(\frac{t-\tilde{z}_{0}}{t-z_{0}}\right)^{\kappa}\left(c_{1} g_{2}-c_{2} g_{1}\right) \mathrm{d} t}{\left(\omega_{1} g_{2}-\omega_{2} g_{1}\right) Y^{+}(t)(t-z)} .
\end{aligned}
$$

When $\kappa \leq-2, Y(z)$ has singularity of order $-\kappa$ at $\tilde{z}_{0}$. Now we aim to cancel the singularity of $\Phi_{0}(z)$ at $\tilde{z}_{0}$. From [5], we know that R problem (10)' is solvable in $R_{0}$ if and only if

$$
\int_{L} \frac{\left(c_{1} g_{2}-c_{2} g_{1}\right) \mathrm{d} t}{\left(\omega_{1} g_{2}-\omega_{2} g_{1}\right) Y^{+}(t)\left(t-\tilde{z}_{0}\right)^{j}}=0, j=2,3, \cdots,-\kappa
$$

and its unique solution takes the form

$$
\begin{aligned}
\Phi_{1}(z)= & \frac{Y(z)}{2 \pi \mathrm{i}} \int_{L} \frac{\left(c_{1} g_{2}-c_{2} g_{1}\right) \mathrm{d} t}{\left(\omega_{1} g_{2}-\omega_{2} g_{1}\right) Y^{+}(t)(t-z)} \\
& -\frac{Y(z)}{2 \pi \mathrm{i}} \int_{L} \frac{\left(c_{1} g_{2}-c_{2} g_{1}\right) \mathrm{d} t}{\left(\omega_{1} g_{2}-\omega_{2} g_{1}\right) Y^{+}(t)\left(t-\tilde{z}_{0}\right)}
\end{aligned} .
$$

For the case $\kappa \leq-2$, since the solution for (10)' in $R_{0}$ is unique and $\tilde{\Phi}_{1}(z)$ must be a solution of (10)' in $R_{0}$, we conclude that $\tilde{\Phi}_{1}(z)=\Phi_{1}(z)$, thus (33) is actually the unique solution of nonhomogeneous problem (8).

Combining the particular solution of nonhomogeneous problem (8) and the general solution of homogeneous problem (14), we known that when $\kappa \geq 0$, the general solution of $\mathrm{R}$ problem (8) is

$$
\Phi(z)=X(z) P_{\kappa}(z)+\Phi_{0}(z)
$$

where $P_{\kappa}(z)$ satisfies condition (24) and $\Phi_{0}(z)$ is given by (31); when $\kappa \leq-2, \mathrm{R}$ problem (8) is solvable if and only if (32) is satisfied and the unique solution is given by (33).

Putting the solution $\Phi(z)$ into the first equation in (1), we obtain

$$
\Psi(t)=\frac{2 \operatorname{Re}\left[\omega_{1}(t) \Phi^{+}(t)\right]-c_{1}(t)}{g_{1}(t)}, t \in L .
$$

Therefore, we derive the following results.

Theorem 5.2. If $\kappa \geq 0$, the nonhomogeneous problem (1) is always solvable and its general solution is $(\Phi(z), \Psi(t))$, where $\Phi(z)$ is given by (34) with $P_{\kappa}(z)$ satisfying condition (24) and $A$ being given by (22) (a real constant factor is permitted for $A$ ), while $\Psi(t)$ is given by (35). If $\kappa \leq-2$, under the necessary and sufficient condition (32), the nonhomogeneous problem (1) has unique solution $(\Phi(z), \Psi(t))$, where $\Phi(z)$ and $\Psi(t)$ are given by (33) and (35) respectively.

\section{REFERENCES}

[1] M. B. Balk, "Polyanalytic Functions," Akademie Verlag, Berlin, 1991.

[2] H. Begehr and A. Kumar, "Boundary Value Problems for the Inhomogeneous Polyanalytic Equation I," Analysis: International Mathematical Journal of Analysis and Its Application, Vol. 25, No. 1, 2005, pp. 55-71.

[3] D. Jinyuan and W. Yufeng, "On Boundary Value Problems of Polyanalytic Functions on the Real Axis," Complex Variables, Vol. 48, No. 6, 2003, pp. 527-542. doi:10.1080/0278107031000103412

[4] B. F. Fatulaev, "The Main Haseman Type Boundary Value Problem for Metaanalytic Function in the Case of Circular Domains," Mathematical Modelling and Analysis, Vol. 6, No. 1, 2001, pp. 68-76. 
[5] J. K. Lu, "Boundary Value Problems for Analytic Functions," World Scientific, Singapore, 1993.

[6] A. S. Mshimba, "A Mixed Boundary Value Problem for Polyanalytic Function of Order $\mathrm{n}$ in the Sobolev Space Wn, p(D)," Complex Variables, Vol. 47, No. 12, 2002, pp. 278-1077.

[7] N. I. Muskhelishvili, "Singular Integral Equations," World Scientific, Singapore, 1993.

[8] W. Yufeng and D. Jinyuan, "Hilbert Boundary Value Problems of Polyanalytic Functions on the Unit Circumference," Complex Variables and Elliptic Equations, Vol. 51, No. 8-11, 2006, pp. 923-943. doi:10.1080/17476930600667692

[9] L. Xing, "A Class of Periodic Riemann Boundary Value Inverse Problems," Proceedings of the Second Asian
Mathematical Conference, Nakhon Ratchasima, 1995, pp. 397-400.

[10] M. H. Wang, "Inverse Riemann Boundary Value Problems for Generalized Analytic Functions," Journal of Ningxia University of Natural Resources and Life Sciences Education, Vol. 27, No. 1, 2006, pp. 18-24.

[11] X. Q. Wen and M. Z. Li, "A Class of Inverse Riemann Boundary Value Problems for Generalized Holomorphic Functions," Journal of Mathematical, Vol. 24, No. 4, 2004, pp. 457-464.

[12] L. X. Cao, P.-R. Li and P. Sun, "The Hilbert Boundary Value Problem With Parametric Unknown Function on Upper Half-Plane," Mathematics in Practice and Theory, Vol. 42, No. 2, 2012, pp. 189-194. 